\title{
The Sister Mary Joseph's Nodule
}

Bzikha Ilham $^{1 *}$, Dkhissi Yassine², Mouaqit Ouadii², Benjelloun El Bachir ${ }^{2}$, Ousadden Abdelmalek², Ait Taleb Khalid², El Bouhaddouti Hicham ${ }^{2}$

${ }^{1}$ Department of General Surgery, CHU Hassan II FES, 30000, Morocco
${ }^{2}$ Department of General Surgery A, CHU Hassan II FES, 30000, Morocco

DOI: $10.36347 /$ sasjs.2021.v07i04.005

| Received: 27.02.2021 | Accepted: 11.03.2021 | Published: 08.04.2021

*Corresponding author: Bzikha Ilham

\section{Abstract}

Sister Mary Joseph nodule or Sister Mary Joseph Sign refers to a palpable nodule bulging into the umbilicus as a result of metastasis of a malignant cancer in the pelvis or abdomen. The Sister Mary Joseph, was the first person who observed that the patients with advanced abdominal-pelvic malignancies, frequently presented a periumbilical palpable nodule. Here, we present a case of Sister Mary Joseph nodule as a gastric cancer metastasis, its diagnosis, treatment and follow-up.

Keywords: Umbilical Nodule, Metastasis, Gastric cancer.

Copyright (C) 2021 The Author(s): This is an open-access article distributed under the terms of the Creative Commons Attribution 4.0 International License (CC BY-NC 4.0) which permits unrestricted use, distribution, and reproduction in any medium for non-commercial use provided the original author and source are credited.

\section{INTRODUCTION}

Sister Mary Joseph nodule $(\mathrm{SMJN})$ is a rare umbilical lesion resulting from an intra-abdominal and/or pelvic malignancy. It was named after Sister Mary Joseph, a surgical assistant to Dr. William J. Mayo, who noted the association between the presence of an umbilical nodule and an intra-abdominal malignancy. Its incidence is $1 \%-3 \%$ of all intraabdominal or pelvic malignancies. Gastrointestinal malignancies, most commonly gastric, colon, and pancreatic, account for about $52 \%$ of cases and gynecological cancers, most commonly ovarian and uterine, account for about $28 \%$ of the underlying sources. Also, 15-29\% of all cases have an unknown origin. The mechanism of tumor spread to the umbilicus is poorly understood as it seems to be lymphatic, vascular, contiguous, or via embryologic remnants in the abdominal wall.

\section{CASE REPORT}

A 60-year-old woman with umbilical tumor presented to our hospital for a surgery consultation. She had a past history of gastric ulcer. Her family history was not relevant. Physical examination showed a $3 \mathrm{~cm}$ firm, protuberant, ulcerated umbilical nodule (Figure1). On palpation a firm liver edge $3 \mathrm{~cm}$ below the right costal margin and percussion of the abdomen revealed ascitis.

Biopsy of the nodule was effectuated for a definitive diagnosis and the anatomopathological study has shown that it's a gastric adenocarcinoma.
CT scan of the abdomen revealed gastric tumor with metastatic lesions and carcinoma (Figure-2). The gastrointestinal endoscopy was not realized.

The patient has received palliative chemotherapy and the evolution was marked by the death of the patient 7 months after.

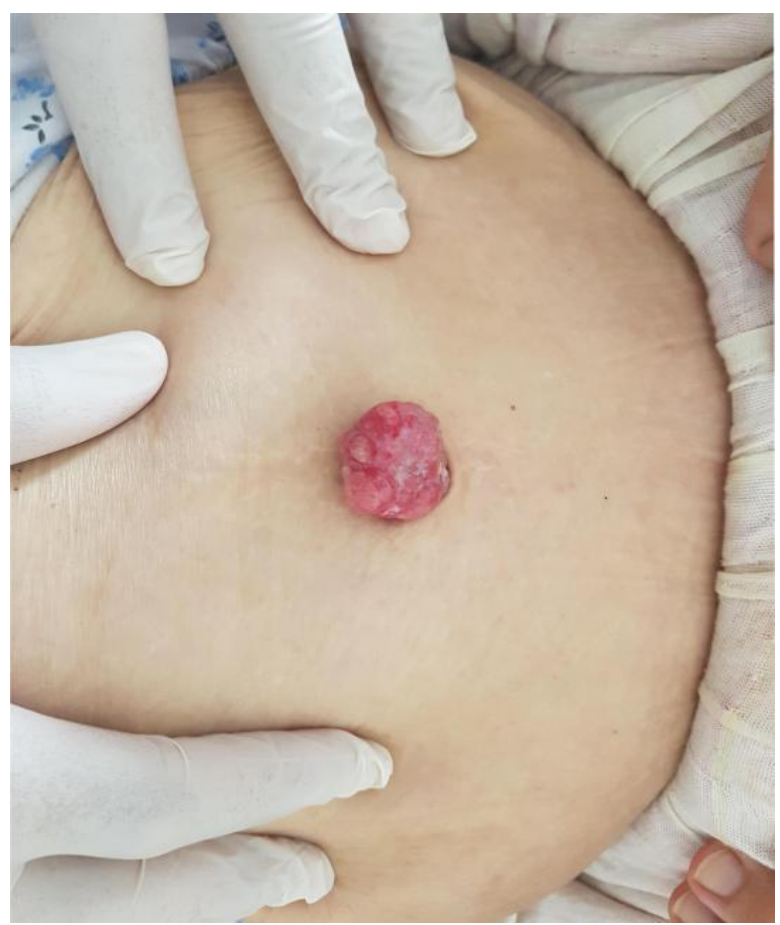

Fig-1: A $3 \mathrm{~cm}$ firm, protuberant, ulcerated tumor was observed in the umbilicus 


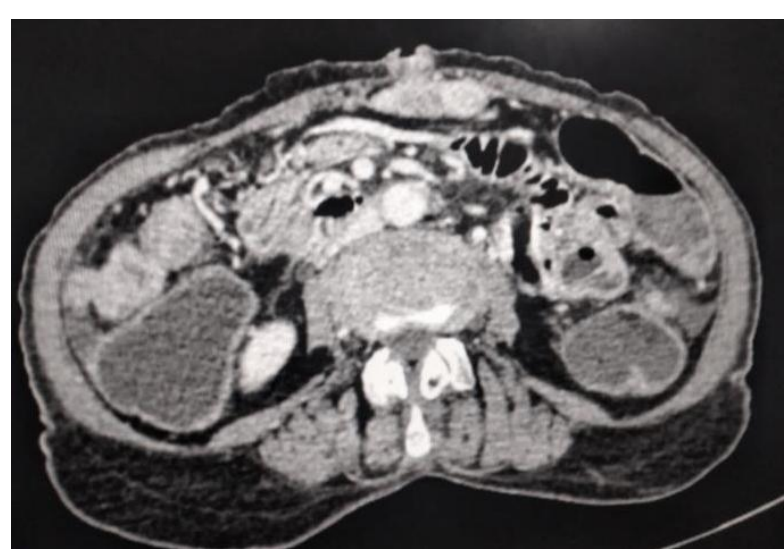

Fig-2: CT scan

\section{DISCUSSION}

Umbilical tumors are rare and can be classified as benign or malignant. Benign causes include umbilical hernia, granuloma, abscess, mycosis, and eczema. Malignant tumors can be either primary or metastatic. The presentation of a SMJN can be quite variable ranging from a hard and irregular nodule to a soft and painfully ulcerated mass. On physical examination, its appearance is often misleading because the skin overlying the lesion can be normal or erythematous. Previous reports showed that $60 \%$ of umbilical nodules were benign. As a result, an umbilical nodule may be present for several months before the diagnosis of a malignancy is finally established [1].

When an umbilical nodule is found it is necessary to make an accurate histological diagnosis between primary and metastatic lesions. Fine needle aspiration cytology and core biopsy are proposed as simple, fast, accurate, and inexpensive diagnostic tools. Also, ultrasonographical study showed a solid hypoechoic mass in the umbilicus with irregular margins and without any signs of inflammation involving the adjacent tissue might suggest the diagnosis of a SMJN.

The most common origins of Sister Mary Joseph nodule are gastrointestinal (52\%), gynecologic (28\%), stomach (23\%) and ovarian (16\%) carcinomas. About $15-29 \%$ of all cases have an unknown origin (as depicted by the presented case) and $3 \%$ originate from the thoracic cavity. Primary tumors in many other sites like gall bladder, uterus, liver, endometrium, small intestine, fallopian tube, appendix, cervix, penis, prostrate, urinary bladder, breast, lung and kidneys have also been reported to cause Sister Mary Joseph nodules. Histology of the metastatic umbilical tumor usually reveals adenocarcinoma but rare reports of umbilical metastasis from sarcomas, mesotheliomas and melanomas have also been seen [2-4]. CT Scan (abdomen and chest) and Fine Needle Aspiration Cytology (FNAC) of the tumor are invaluable in the diagnosis of Sister Mary Joseph nodule and help to exclude a primary benign umbilical neoplasm. In 14$33 \%$ of cases, umbilical metastases lead to the diagnosis of previously occult neoplasms [5]. In $40 \%$ of patients with a known neoplasm the nodule was an early sign of relapse [6]. Spread of metastatic carcinoma to the umbilical region has been hypothesized to occur by either contiguous spread of peritoneal cancer, hematogenous spread through arterial and venous systems or lymphatic spread (mainly pancreatic carcinoma) with extension along ligaments of embryonic origin (round ligament of liver, urachus, vitello intestinal duct reminant and the obliterated vitelline artery).

Previous studies showed that, among umbilical malignancies, $88 \%$ originated outside the umbilicus and $12 \%$ were primary skin tumors. The mean age of diagnosis is approximately 50 years, with a range of 18-87 years. Moreover, women are more likely to have malignant tumors affecting the umbilicus. Although SMJN is most commonly associated with gastrointestinal malignancies, in this case both upper and lower endoscopies did not provide major findings. Histologically, a metastatic umbilical tumor usually reveals an adenocarcinoma; however, sarcomas, mesotheliomas, and melanomas have also been reported. In this case the final pathological diagnosis was an ovarian serous adenocarcinoma with intraabdominal and thoracic metastases.

SMJN is considered a late manifestation of a malignant process and represents an advanced stage of the disease. Mean life expectancy is $2-11$ months without treatment. Recent reports have proposed an aggressive treatment combining surgical excision, radiotherapy, and chemotherapy with a mean survival of 6-21 months. However, as the disease is usually advanced and metastatic, often only palliative treatment is offered. The patient in this case received surgery treatment and is still undergoing chemotherapy without complications. CA125 levels have been decreasing which made us suspect a good response for the treatment; however, strict follow-up is necessary [7].

\section{CONCLUSION}

In conclusion, the presence of SMJN is a rare and often poor prognostic sign of a disseminated malignancy. SMJN needs to be considered as a differential diagnosis of an umbilical nodule in order to make a prompt identification of the primary lesion.

Conflicts of interest: The authors certify that there is no conflict of interest with any financial organization regarding the material discussed in the manuscript.

Funding: The authors report no involvement in the research by the sponsor that could have influenced the outcome of this work.

Ethics Approval: IRB and clinical trial registration approval are not required for case reports. 
Constant of the use of medical data is obtained verbally from the patient.

\section{REFERENCES}

1. Salemis NS. Umbilical metastasis or Sister Mary Joseph's Nodule as a very early sign of an occult cecal adenocarcinoma. J Gastrointest Canc. 2007;38(2-4):131-134.

2. Touraud JP, Lentz N, Dutronc Y, Mercier E, Sagot $\mathrm{P}$, Lambert D. Umbilical cutaneous metastasis (or Sister Mary Joseph's nodule) disclosing an ovarian adenocarcinoma. Gynecologie, obstetrique \& fertilite. 2000 Oct 1;28(10):719-21.

3. Piura B, Meirovitz M, Bayme M, Shaco-Levy R. Sister Mary Joseph's nodule originating from endometrial carcinoma incidentally detected during surgery for an umbilical hernia: a case report. Arch Gynecol Obstet. 2006;274(6):385-88.

4. Sharma A, Sharma V. Image Diagnosis: Sister Mary Joseph nodule. Perm J. 2014;18(2):e132e132.

5. Quenu L. Du cancer secondaire de l'ombilic. Secondary cancer in the umbilicus Rev Chir. 1896;16:97-133.

6. Galvan VG. Sister Mary Joseph's nodule. Ann Intern Med. 1998;128(5):410.

7. Powell FC, Cooper AJ, Massa MC, Goellner JR, Su WP. Sister Mary Joseph's nodule: a clinical and histologic study. J Am Acad Dermatol. 1984;10(4):610-5. 